\title{
Экспериментальное и теоретическое исследования характеристик позиционно-чувствительных фотоприемников на основе эпитаксиальных слоев $\boldsymbol{n}$-CdSe/слюда
}

\author{
(С) Э.А. Сенокосов ${ }^{1}$, В.И. Чукита ${ }^{1}$, Р.А. Хамидуллин ${ }^{1}$, В.Н. Чебан ${ }^{1}$, И.Н. Один, ${ }^{2,}$, М.В. Чукичев \\ ${ }^{1}$ Приднестровский государственный университет им. Т.Г. Шевченко, \\ MD-3300 Тирасполь, Приднестровская Молдавская Республика \\ ${ }^{2}$ Московский государственный университет им. М.В. Ломоносова, \\ 119991 Москва, Россия \\ I E-mail: i.n.odin@mail.ru
}

(Получена 15 сентября 2016 г. Принята к печати 2 ноября 2016 г.)

\begin{abstract}
Приведены экспериментальные результаты исследования выходных характеристик четырехконтактных полупроводниковых позиционно-чувствительных фотоприемников, изготовленных на основе эпитаксиальных фоточувствительных слоев $n$-CdSe/слюда. Проведен теоретический анализ позиционной чувствительности слоев на основе элементарной теории токопротекания и модели электрического диполя. Установлено, что теоретические характеристики координатной чувствительности исследованных позиционно-чувствительных фотоприемников коррелируют с экспериментальными зависимостями как по форме кривых, так и положению максимумов. Результаты определения удельной спектральной чувствительности свидетельствуют о перспективности слоев $n$-CdSe в качестве позиционно-чувствительных фотоприемников.
\end{abstract}

DOI: 10.21883/FTP.2017.05.44430.8406

\section{1. Введение}

Позиционно-чувствительные фотоприемники используются для обнаружения источника излучения, бесконтактного определения расстояния, для прецизионного определения координат облучаемой области в режиме реального времени, а также слежения за движущимися объектами $[1,2]$. К настоящему времени разработаны и используются позиционно-чувствительные фотоприемники на основе $p-n$-переходов $[1,2]$, фоторезистивных слоев, металл-оксид-полупроводниковых (МОП) транзисторов [3]. Однако функциональные фоторезисторы имеют сравнительно низкую разрешающую способность, а технология производства МОП-транзисторов и материалов с $p-n$-переходами, используемых для позиционночувствительных фотоприемников, сложна. Кроме того, указанные фотоприемники обладают чувствительностью к свету лишь в области собственного поглощения светоприемного элемента.

В работах [4,5] впервые предложена конструкция полупроводниковых позиционно-чувствительных фотоприемников (ППЧФ). В них приемным элементом являются однородные слои полупроводника, которые позволяют фиксировать местоположение излучающего объекта по одной или двум координатам, а спектральная область их чувствительности может быть расширена за счет примесной фотопроводимости $[4,5]$. В качестве материала для ППЧФ были исследованы слои теллурида кадмия [6]. Среди проблем создания ППЧФ следует отметить необходимость разработки полупроводниковых материалов с более высокой фоточувствительностью, чем у теллурида кадмия, например, слоев селенида кадмия. Требуется экспериментальное и теоретическое исследование новых материалов для ППЧФ, в особенности слоев $\mathrm{CdSe}$ с высокой фоточувствительностью.
Цель настоящей работы заключается в экспериментальном и теоретическом исследованиях выходных характеристик ППЧФ, изготовленных на основе эпитаксиальных фотопроводящих слоев $n$-CdSe. Теоретический анализ проведен как на основе элементарной теории токопротекания, так и с использованием модели электрического диполя; проведено сравнение рассчитанных данных с экспериментальными результатами.

\section{2. Методика эксперимента}

\section{1. Получение и свойства эпитаксиальных слоев $n$-CdSe/слюда}

Специально не легированные слои CdSe выращены на кристаллических подложках слюды (мусковит) методом термического испарения в вакууме в квазизамкнутом объеме, как описано в работе [7]. Выращены эпитаксиальные слои $\mathrm{CdSe}$ со структурой вюртцита, ориентированные плоскостью (0001) параллельно плоскости подложки. Слои обладали высоким структурным совершенством, поверхность их была зеркально-гладкой. Слои выращивали в форме диска радиусом $R=9$ мм и толщиной $d=15-50$ мкм. Слои CdSe имели $n$-тип проводимости и были фоточувствительными - значения кратности фотоотклика при освещении интегральным светом 200 лк находились в пределах $1 \cdot 10^{4}-3 \cdot 10^{4}$.

\section{2. Методика исследования характеристик позиционной чувствительности эпитаксиальных слоев $\boldsymbol{n}$-CdSe}

В работе исследовали выходные характеристики четырехконтактных ППЧФ, изготовленных на основе фото- 
проводящих эпитаксиальных слоев $n$-CdSe. На боковые поверхности слоев $n$-CdSe наносили индиевые контакты, располагаемые в вершинах квадрата $A B C D$ - схема расположения контактов приведена на рис. 1 . К контактам $A, C$ подключали источник входного напряжения $U_{A C}$, и через фотоприемный элемент пропускали ток $I_{m}$. К контактам $B, D$, расположенным на эквипотенциальной линии тока, с сопротивления нагрузки $R_{n}$ снимали выходное напряжение $U_{B D}$, возникающее при локальной засветке лучом зонда поверхности приемного элемента ППЧФ. Оптическое зондирование поверхности $\mathrm{CdSe}$ осуществляли лучом твердотельного лазера марки $\mathrm{LD}-67005 \mathrm{~W}$ с длиной волны 670 нм и средней мощностью 5 мВт. Луч лазера направляли перпендикулярно поверхности фотоприемного элемента и фокусировали в пятно радиусом $а$ около $0.5 \mathrm{MM}$.

Появление напряжения $U_{B D}$ происходит вследствие разбаланса цепи интегрального электрического моста, в четырех плечах которого до освещения находились пространственно распределенные сопротивления одинаковой величины. При засветке полупроводникового слоя ППЧФ генерируются избыточные носители тока. В результате этого уменьшается сопротивление освещенного плеча фотоприемного элемента, что вызывает разбаланс моста и появление разности потенциалов $U_{B D}$. По величине и полярности напряжения $U_{B D}$ судили о позиционной чувствительности фотоприемника.

В общем случае выходное напряжение четырехконтактных ППЧФ на основе $n$-CdSe зависело как от местоположения и интенсивности светового пятна, так и от величины тока $I_{\text {in }}$.

Близкая к квадратной симметрии зависимость выходного сигнала $U_{B D}$ показывает, что в качестве равноправного приемного элемента может быть выбран любой из четырех его квадрантов.

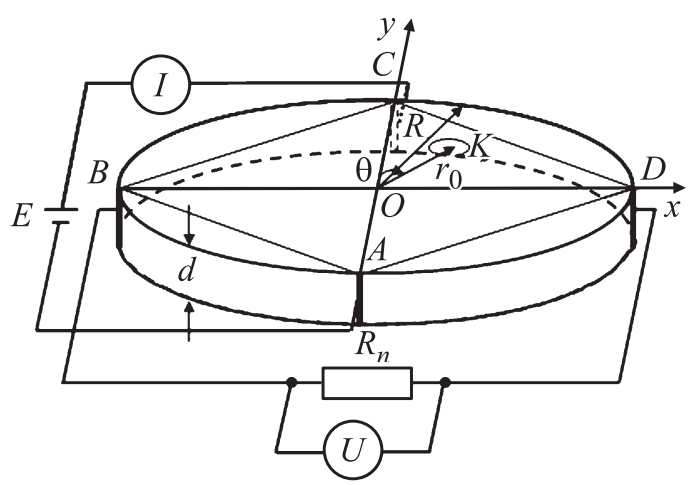

Pис. 1. Схема расположения контактов и светового пятна на поверхности приемного элемента ППЧФ $n$-CdSe при оптическом зондировании. Обозначения: $R-$ радиус диска CdSe c толщиной $d ; A, B, C, D-$ индиевые контакты; $O-$ начало координат; $x, y-$ оси координат; $K-$ пятно засветки; $r_{0}-$ радиус окружности сканирования; $\theta-$ угол между осью $y$ и прямой, проведенной из начала координат в центр пятна засветки; $R_{n}-$ сопротивление нагрузки; $E-$ источник напряжения; $I, U-$ приборы для измерения тока и напряжения.

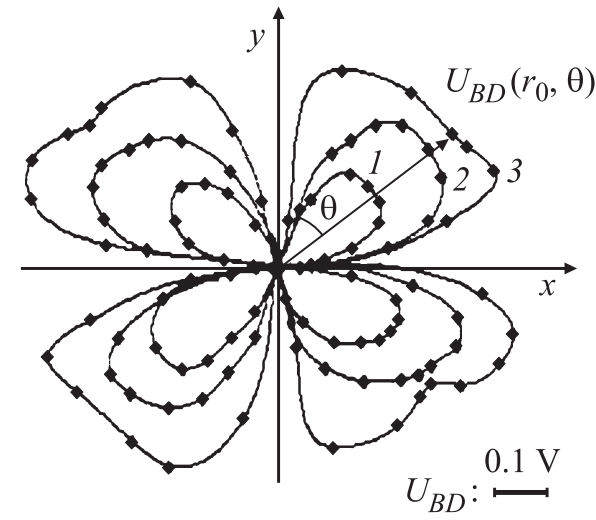

Рис. 2. Полярные диаграммы экспериментальной зависимости выходного напряжения $U_{B D}$ четырехконтактного ППЧФ $n$-CdSe от координат пятна засветки для радиусов $r_{0}: 1-0.3 R$, $2-0.5 R, 3-0.7 R ; \theta-$ угол между осью $y$ и прямой, проведенной из начала координат в центр области засветки.

\section{3. Экспериментальные результаты}

На рис. 2 приведены полярные диаграммы выходного напряжения $U_{B D}\left(r_{0}, \theta\right)$ четырехконтактных ППЧФ $n$-CdSe. Полярные диаграммы получены при температуpe $298 \mathrm{~K}$, входном токе $I_{\text {in }}=2.0$ мкА, мощности оптического зонда 3 мВт и трех значениях радиуса $r_{0}$.

В каждом квадранте напряжение $U_{B D}$ возрастает по абсолютной величине с ростом $r_{0}$. Напряжение $U_{B D}$ равно нулю для точек, расположенных на осях $x$ и $y$. В случае $r_{0}<0.7 R$ максимальное значение выходного напряжения $U_{B D}$ достигается при положениях пятна засветки на биссектрисах углов между диагоналями квадрата (рис. 2, кривые 1,2). В каждом квадранте диаграммы выходного напряжения при $r_{0}=0.3 R$ или $0.5 R$ фигуры имеют однолепестковую форму (рис. 2, кривые 1,2 ), как и при аналогичном изучении слоев $n$-CdTe [6].

При оптическом зондировании в случае $r_{0} \geq 0.7 R$ на полярных диаграммах выходного напряжения в каждом квадранте приемного элемента появляется по два максимума (рис. 2, кривая 3) - образуются двухлепестковые фигуры, как и при исследовании слоев $n$-CdTe [6].

Другие экспериментальные результаты изложены в разд. 4.2, 4.3, 4.5 при сравнении с рассчитанными данными.

\section{4. Теоретический анализ позиционной чувствительности ППЧФ $n$-CdSe}

\section{1. Анализ позиционной чувствительности ППЧФ на основе элементарной теории токопротекания}

В соответствии с элементарной теорией токопротекания, развитой на основе решения уравнения Лапласа [8], 


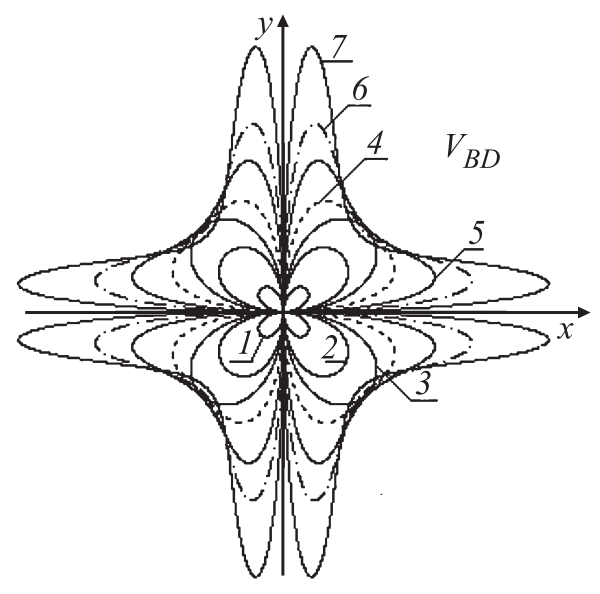

Рис. 3. Полярные диаграммы теоретической зависимости выходного сигнала четырехконтактного ППЧФ $n$-CdSe при оптическом зондировании для значений безразмерных радиусов $r_{00}: 1-0.3,2-0.5,3-0.6,4-0.7,5-0.8,6-0.85$, $7-0.9$.

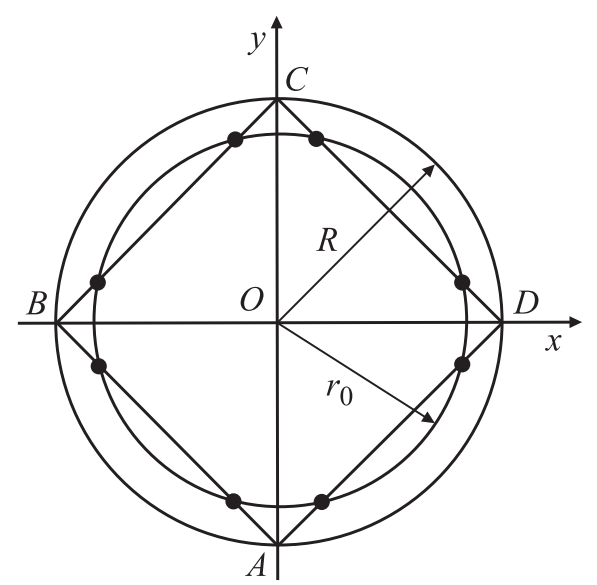

Рис. 4. Точки пересечения окружности зондирования со сторонами квадрата $A B C D$ на схеме расположения контактов в случае $r_{00} \geq 0.7$.

выходной сигнал напряжения $U_{B D}$ исследуемых ППЧФ зависит от величины входного тока $I_{\text {in }}$, от радиуса светового пятна и его местоположения, от электропроводности и ее увеличения при засветке, от величины входного тока $I_{i n}$. Согласно [8] выходное напряжение $U_{B D}$ определяется выражением

$$
U_{B D}=\frac{\delta \sigma}{\sigma} \frac{a^{2}}{R^{2}} \frac{I_{i n}}{\pi \sigma d} \frac{8 r_{00}^{2} \sin 2 \theta}{\left(1-r_{00}^{4}\right)^{2}+4 r_{00}^{4} \sin ^{2} 2 \theta},
$$

где $\sigma-$ удельная электропроводность диска $\mathrm{CdSe}$; $\delta \sigma$ - изменение $\sigma$ при локальной засветке, $a-$ радиус пятна засветки; $I_{\text {in }}-$ входной ток; $\theta-$ угол между вектором $\mathbf{r}_{0}$ и положительным направлением оси $y ; R$ - радиус диска CdSe с толщиной $d ; r_{0}-$ радиус окружности зондирования (расстояние от начала координат до центра светового пятна $K) ; r_{00}=r_{0} / R$.
На рис. 3 представлены рассчитанные по формуле (1) зависимости безразмерной разности потенциалов $V_{B D}=\frac{U_{B D}}{N}\left(\right.$ где $\left.N=\frac{\delta \sigma a^{2} I_{i n}}{R^{2} \pi \sigma^{2} d}\right)$ от координат пятна засветки для различных значений безразмерного радиуса $r_{00}$. При засветке точек приемного элемента, находящихся на осях $x$ и $y$, выходной сигнал равен нулю. При построении диаграмм на радиус-векторах, проведенных из начала координат под углом $\theta$, откладывали рассчитанные значения потенциала $V_{B D}$.

Диаграммы чувствительности при $r_{00}<0.7$ представляют собой систему однолепестковых фигур (рис. 3; кривые $1-3)$. Для них максимумы функции $V_{B D}(\theta)$ лежат на биссектрисах углов между диагоналями квадрата.

При $r_{00} \geq 0.7$ полярные диаграммы чувствительности ППЧФ представляют собой систему симметричных двухлепестковых фигур (рис. 3, кривые 4-7). В этом случае максимумы функции $V_{B D}(\theta)$ соответствуют точкам пересечения окружности зондирования со сторонами квадрата $A B C D$, эти точки изображены на рис. 4. С увеличением $r_{00}$ максимумы $V_{B D}(\theta)$ функции смещаются к ближайшим осям $x$ и $y$ (рис. 3 , кривые $4-7$ ).

\section{2. Сопоставление экспериментальных диаграмм позиционной чувствительности с данными, рассчитанными на основе элементарной теории токопротекания}

Сопоставление экспериментальных полярных диаграмм чувствительности ППЧФ $n$-CdSe (рис. 2) с рассчитанными по формуле (1) (рис. 3) свидетельствует о корреляции кривых по форме и положению максимумов при одинаковом значении $r_{00}$. И в эксперименте, и в расчетах при $r_{00}<0.7$ образуются однолепестковые фигуры, а при $r_{00} \geq 0.7$ - двухлепестковые фигуры.

На рис. 5 (кривая 1) показана экспериментальная зависимость выходного сигнала от радиуса $r_{0}$ при оптическом зондировании фотоприемника CdSe. Кривая снята при температуре $298 \mathrm{~K}$, мощности зонда $3 \mathrm{MBT}$, значении входного тока $I_{i n}=2.0$ мкА и значении угла $\theta$, равном $\pi / 4$. Далее, по формуле (1) провели расчеты

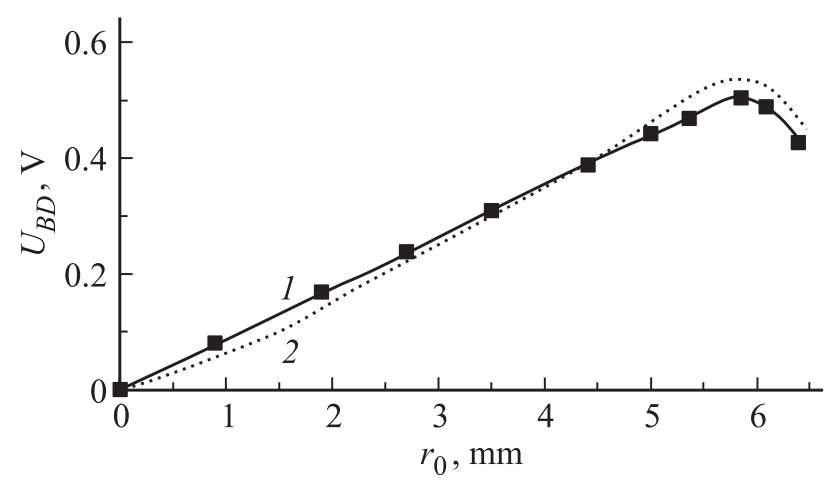

Рис. 5. Экспериментальная (1) и теоретическая (2) зависимости напряжения $U_{B D}$ от радиуса $r_{0}$ при оптическом зондировании фотоэлемента $n$-CdSe. 
$U_{B D}=f\left(r_{0}\right)$. Видно, что экспериментальная зависимость $U_{B D}=f\left(r_{0}\right) \quad$ (рис. 5, кривая 1 ) хорошо коррелирует с кривой, рассчитанной по формуле (1) (рис. 5, кривая 2). Экспериментальная зависимость проходит через максимум при $r_{0}=5.85 \mathrm{MM}=0.65 R$, соответствующий также и максимальному значению $U_{B D}$ на рассчитанной кривой.

\section{3. Экспериментальные результаты определения спектральной и координатной чувствительности слоев $n$-CdSe}

Для возрастающего участка экспериментальной зависимости $U_{B D}=f\left(r_{0}\right)$ (рис. 5, кривая 1) при токе $I_{\text {in }}=2.0$ мкА определена удельная спектральная чувствительность фотоприемников $n$-CdSe, которая составляет $80 \mathrm{MB} /($ мкА - мВт). При равных условиях возбуждения она в 12 раз превышает чувствительность ППЧФ $n$-СdTе [6], что свидетельствует о несомненной перспективности ППЧФ на основе слоев селенида кадмия. Найдено также, что удельная координатная чувствительность ППЧФ $n$-CdSe составляет $150 \mathrm{MB} /(\mathrm{Mм} \cdot \mathrm{м \kappa А} \cdot \mathrm{MBT})$. Это представляет интерес для дальнейшего исследования ППЧФ на основе эпитаксиальных слоев $\mathrm{CdSe}$ в связи с определением координат источников света.

\section{4. Теоретический анализ позиционной чувствительности ППЧФ на основе модели электрического диполя}

Физическая природа чувствительности ППЧФ объясняется влиянием дипольного момента, образующегося в области локального оптического возбуждения светоприемного элемента под действием сил внешнего электрического поля. При этом дипольный момент вносит основной вклад в выходной сигнал ППЧФ [9]. В этой области генерируются избыточные носители тока с концентрацией $\Delta n$. Под действием сил внешнего электрического поля электроны смещаются против этих сил на расстояние $\mathbf{v}_{e} \tau_{e}$, а дырки - на расстояние $\mathbf{v}_{h} \tau_{h}$ по направлению сил электрического поля. Здесь $\mathbf{v}_{e}, \mathbf{v}_{h}$ и $\tau_{e}, \tau_{h}$ - дрейфовые скорости и времена жизни фотоэлектронов и фотодырок соответственно. В результате этого на одной стороне области засветки образуется нескомпенсированный отрицательный заряд, а на противоположной - нескомпенсированный положительный заряд. В общем случае диаметр области локального оптического возбуждения значительно меньше расстояния от нее до электрических контактов. Поэтому в мультипольном разложении потенциала области локального оптического возбуждения можно ограничиться дипольным приближением. Потенциал электрического диполя [10] в точке пространства $\left(x_{1}, y_{1}\right)$ равен:

$$
\phi=\frac{1}{\varepsilon} \frac{\left(\mathbf{P r}_{1}\right)}{r_{1}^{3}},
$$

где $\mathbf{P}$ - дипольный момент области локального оптического возбуждения, $\varepsilon$ - относительная диэлектрическая проницаемость полупроводника, $\mathbf{r}_{1}\left(x_{1}, y_{1}\right)$ - радиусвектор, отсчитываемый от центра диполя. Выражение для темнового потенциала фотоприемника при протекании вдоль него электрического тока $I_{\text {in }}$ имеет вид [9]

$$
\phi_{0}=\frac{I_{\text {in }}}{\sigma_{0} \pi d} \ln \left|\frac{r-R_{y}}{r+R_{y}}\right|,
$$

где $\sigma_{0}$ - удельная темновая электропроводность, $\mathbf{r}-$ радиус-вектор рассматриваемой точки; $\mathbf{R}_{y}=R \mathbf{e}_{y}$, где $\mathbf{e}_{y}$ - единичный вектор вдоль оси $y$.

Потенциал, создаваемый диполем вблизи боковой поверхности полупроводникового диска $n$-CdSe при условиях $a \ll R, a \ll l_{0} R, \frac{r_{1} l_{0}}{a^{2}} \gg 1$, описывается выражением

$$
\begin{aligned}
\phi\left(r_{1}\right)= & \frac{\pi e \Delta n a^{2}(1-\exp (-\alpha d))}{\varepsilon \alpha} \frac{r_{1} l_{0}}{r_{1}^{3}} \\
& \times\left[1-\left(\frac{3 l_{0}^{2}+d^{2}}{8 r_{1}^{2}}\right)+\frac{15}{4} \frac{\left(r_{1} l_{0}\right)^{2}}{r_{1}^{4}}\right],
\end{aligned}
$$

где $e-$ элементарный электрический заряд, $\alpha-$ коэффициент поглощения света для полупроводника, $l_{0}=\left|\mathbf{l}_{0}\right|=\left|\mathbf{v}_{e} \tau_{e}+\mathbf{v}_{h} \tau_{h}\right|-$ модуль вектора смещения облака неравновесных электронов относительно облака неравновесных дырок под действием внешнего электрического поля $\mathbf{E}=-$ grad $\phi_{0}$. Для точек на боковой поверхности диска второе и третье слагаемые в квадратных скобках формулы (4) значительно меньше единицы. Если падающий свет полностью поглощается слоем полупроводника, т.е. когда $\exp (-\alpha d) \ll 1$, выражение (4) принимает вид

$$
\phi\left(\mathbf{r}_{1}\right)=\frac{\pi e \Delta n a^{2}}{\varepsilon \alpha} \frac{\mathbf{r}_{1} \mathbf{l}_{0}}{r_{1}^{2}} .
$$

Сравнивая (2) и (5), получаем уравнение для дипольного момента:

в котором

$$
\mathbf{P}=\frac{\pi e \Delta n a^{2} \mathbf{I}_{0}}{\alpha}=P_{0} \mathbf{E},
$$

$$
P_{0}=\frac{\pi e \Delta n a^{2}}{\alpha}\left(\mu_{e} \tau_{e}+\mu_{h} \tau_{h}\right) .
$$

Здесь $\mu_{e}$ и $\mu_{h}-$ подвижности фотоэлектронов и фотодырок соответственно. С учетом формул (6) и (7) разность потенциалов между электрическими контактами $B$ и $D$ светоприемного элемента будет равна:

$$
\begin{aligned}
U_{B D} & =-\left(\phi_{B}-\phi_{D}\right)=-\frac{\mathbf{P}}{\varepsilon}\left[\frac{\mathbf{r}_{K B}}{r_{K B}^{3}}-\frac{\mathbf{r}_{K D}}{r_{K D}^{3}}\right] \\
& =-\frac{P_{0}}{\varepsilon}\left[\frac{\mathbf{E r}_{K B}}{r_{K B}^{3}}-\frac{\mathbf{E r}_{K D}}{r_{K D}^{3}}\right],
\end{aligned}
$$

где $\mathbf{r}_{K B}$ и $\mathbf{r}_{K D}-$ радиус-векторы, соединяющие точку засветки $K$ с точками $B$ и $D$ соответственно (рис. 1$)$. 


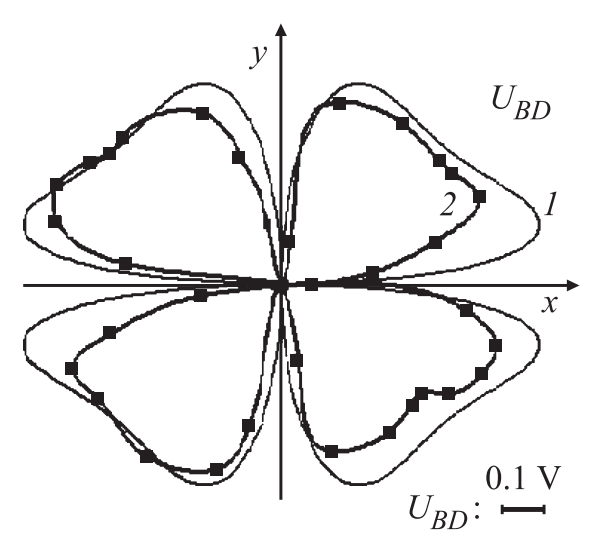

Рис. 6. Полярные диаграммы чувствительности четырехконтактного ППЧФ $n$-CdSe для $r_{00}=0.7: 1-$ рассчитанные по формуле (9) кривые, 2 - экспериментальные кривые.

Учитывая выражение для темнового потенциала (3) и соответствующее ему распределение напряженности внешнего электрического поля $\mathbf{E}=-\operatorname{grad} \phi_{0}$, из формулы (8) получаем выражение для разности потенциалов $U_{B D}$ в модели электрического диполя:

$$
U_{B D}=-\frac{P_{0} I_{i n}}{\pi \varepsilon \sigma_{0} d} M\left(N_{1}-N_{2}\right),
$$

где

$$
\begin{gathered}
M=\frac{2 R r_{0} \sin \theta}{\left[\left(R^{2}+r_{0}^{2}\right)^{2}-4 R^{2} r_{0}^{2} \sin ^{2} \theta\right]} \\
N_{1}=\frac{r_{0}^{2}-R^{2}+2 R r_{0} \cos \theta}{\left[R^{2}+r_{0}^{2}+2 R r_{0} \cos \theta\right]^{3 / 2}} \\
N_{2}=\frac{r_{0}^{2}-R^{2}-2 R r_{0} \cos \theta}{\left[R^{2}+r_{0}^{2}-2 R r_{0} \cos \theta\right]^{3 / 2}}
\end{gathered}
$$

Расчеты выходного напряжения $U_{B D}$ проводили по формуле (9), рассчитанные для $r_{00}=0.7$ фигуры приведены на рис. 6 (кривая 1).

Рост $U_{B D}$ при перемещении пятна засветки от центра фотоприемного элемента $n$-CdSe к периферии вдоль биссектрисы каждого квадранта при $r_{00}<0.7$ обусловлен увеличением модуля вектора $\mathbf{r}_{K B}$ и уменьшением модуля вектора $\mathbf{r}_{K D}$, согласно формуле (8). Это приводит к появлению однолепестковых фигур, аналогичным фигурам 1 и 2 на рис. 2.

Уменьшение темпа роста $U_{B D}$ при $r_{00} \geq 0.7$ связано с увеличением модуля вектора $\mathbf{r}_{K D}$ и переориентацией диполя в области оптического возбуждения фотоприемника $n$-CdSe. Кроме того, при смещении пятна засветки к боковой границе фотоприемного элемента снижается напряженность внешнего электрического поля в диполе, что приводит к спаду зависимости $U_{B D}=f\left(r_{00}\right)$ при $r_{00} \geq 0.7$. Это вызывает появление на теоретической диаграмме чувствительности ППЧФ двухлепестковых фигур (рис. 6, кривая 1).

\section{5. Сопоставление экспериментальной диаграммы позиционной чувствительности с данными, рассчитанными по модели электрического диполя}

Теоретические расчеты координатной чувствительности ППЧФ, проведенные в рамках дипольного представления по формуле (9), коррелируют с экспериментальными данными. Это, в частности, следует из рис. 6, на котором представлены теоретическая диаграмма чувствительности (кривая 1), построенная в соответствии с формулой (9) для $r_{0}=0.7 R\left(r_{00}=0.7\right)$, и экспериментальная диаграмма (кривая 2), снятая для пленочного фотоприемника $n$-CdSe при таком же значении $r_{0}$. И в эксперименте, и в расчете наблюдается образование двухлепестковых фигур, характерных для случая с $r_{0}=0.7 R\left(r_{00}=0.7\right)$.

\section{6. Сопоставление данных, рассчитанных по модели электрического диполя и на основе элементарной теории токопротекания}

Сопоставление рассчитанных полярных диаграмм чувствительности ППЧФ $n$-CdSe по модели электрического диполя (формула (9)) и на основе элементарной теории токопротекания (формула (1)) свидетельствует о том, что они в основном коррелируют между собой по форме и положению максимумов. В расчетах по обеим моделям при $r_{00}<0.7$ образуются однолепестковые фигуры, а при $r_{00} \geq 0.7$ - двухлепестковые фигуры (рис. 3 и 6, кривая 1).

Следует подчеркнуть, что дипольное представление в области локального оптического возбуждения [9] и элементарная теория токопротекания [8] являются предельными случаями одного и того же неравновесного процесса, протекающего в исследованных фотоэлементах. Возникает вопрос о том, какая из этих двух моделей лучше описывает экспериментальные данные. Можно отметить, что формулу (9) следует применять, когда произведение дрейфовых скоростей носителей тока (фотоэлектронов или фотодырок) $\mathbf{v}_{e}, \mathbf{v}_{h}$ на их времена жизни $\tau_{e}, \tau_{h}$ сравнимо с радиусом пятна засветки $a$. Формулу (1) следует применять в случае, когда указанное произведение много меньше радиуса пятна засветки.

\section{5. Заключение}

В работе экспериментально изучены полярные диаграммы чувствительности четырехконтактных ППЧФ, изготовленных на основе эпитаксиальных слоев $n$-CdSe/ слюда.

Проведен теоретический анализ позиционной чувствительности фотоприемников $n$-CdSe как на основе элементарной теории токопротекания, так и на основе 
модели электрического диполя. Установлено, что рассчитанные характеристики координатной чувствительности ППЧФ $n$-CdSe коррелируют с экспериментальными зависимостями по форме кривых и положению максимумов.

Определена удельная спектральная чувствительность фотоприемников $n$-CdSe, которая составляет величину $80 \mathrm{MB} /($ мкА - мВт). При равных условиях возбуждения она в 12 раз превышает чувствительность ППЧФ, изготовленных на основе $n$-CdTe [6], что свидетельствует о несомненной перспективности ППЧФ на основе слоев селенида кадмия. О перспективности слоев $n$-CdSe свидетельствует и высокая удельная координатная чувствительность, что важно для выявления возможностей использования ППЧФ n-CdSe.

\section{Список литературы}

[1] Дж. Фрайден. Современные датчики: Справочник (М., Техносфера, 2005) с. 308. [Пер. с англ. Ю.А. Заболотной под ред. Е.Л. Свинцова: J. Fraden. Handbook of Modern Sensors: Physics, Designs, and Applications (N.Y.-Berlin-Heidelberg-Hong Kong-London-Milan-Paris-Tokio, Springer, 2004).

[2] А.Н. Марченко, С.В. Свечников, А.К. Смовж. Полупроводниковые сенсорные потенциометрические элементы (М., Радио и связь, 1988) с. 5.

[3] H. Niu, C. Aoki, T. Matsuda. Jpn. Appl. Phys., 26 (1), L35 (1987).

[4] Э.А. Сенокосов, А.А. Клюканов, А.Н. Усатый, С.А. Сергеев, В.М. Федоров. А. с. СССР № 1436796, опубликовано 08.07.1988.

[5] А.А. Клюканов, Э.А. Сенокосов, А.Н. Усатый, В.М. Федоров. А.c. СССР № 1499119, опубликовано 07.08.1989.

[6] Э.А. Сенокосов, В.В. Сорочан. Прикл. физика, № 2, 77 (2006).

[7] Э.А. Сенокосов, В.И. Чукита, И.Н. Один, М.В. Чукичев, Е.С. Абрамова. Неорг. матер., 48 (12), 1299 (2012).

[8] А.А. Клюканов, Э.А. Сенокосов, Д.Е. Богинский, В.В. Сорочан, Л.В. Фещенко. Технология и конструирование в электронной аппаратуре, № 1, 49 (2003).

[9] Э.А. Сенокосов, В.В. Сорочан, Р.А. Хамидуллин, Е.И. Брусенская. Вестн. Приднестровского ун-та, № 3, 7 (2009).

[10] Я.П. Терлецкий, Ю.П. Рыбаков. Электродинамика (М., Высш. шк., 1990) с. 48.

Редактор Г.А. Оганесян

\section{Experimental and theoretical studies of the characteristics of the position-sensitive photodetectors on the basis of epitaxial layers of the $n$-CdSe/mica}

\author{
E.A. Senokosov ${ }^{1}$, V.I. Chukita ${ }^{1}$, R.A. Khamidullin ${ }^{1}$, \\ V.N. Cheban ${ }^{1}$, I.N. Odin ${ }^{2}$, M.V. Chukichev ${ }^{2}$ \\ ${ }^{1}$ Shevchenko State University, \\ MD-3300 Tiraspol, Transdniester Moldovian Republic \\ ${ }^{2}$ Lomonosov Moscow State University, \\ 119991 Moscow, Russia
}

Abstract The experimental results of research of the output characteristics of four-contact semiconductor position-sensitive photodetectors made on the basis of epitaxial layers of photosensitive $n$-CdSe/mica are reported. The theoretical analysis of the positional sensitivity of the layers based on the elementary theory of the current flow and the electric dipole model was conducted. It was found that the theoretical characteristics of the sensitivity of the investigated coordinate position-sensitive photodetectors are correlated with their experimental dependences in forms of curves and positions of the maximums. The results of the determination of the specific spectral sensitivity indicate the prospects of layers of $n$-CdSe as a position-sensitive photodetectors. 